6. van de Laar IM, et al. Mutations in SMAD3 cause a syndromic form of aortic aneurysms and dissections with early-onset osteoarthritis. Nat Genet. 2011;43(2):121-126.

7. Regalado ES, et al. Exome sequencing identifies SMAD3 mutations as a cause of familial thoracic aortic aneurysm and dissection with intracranial and other arterial aneurysms. Circ Res. 2011;109(6):680-686.

8. Guo DC, et al. Mutations in smooth muscle alphaactin (ACTA2) lead to thoracic aortic aneurysms and dissections. Nat Genet. 2007;39(12):1488-1493.

9. Zhu L, et al. Mutations in myosin heavy chain 11 cause a syndrome associating thoracic aortic aneurysm/aortic dissection and patent ductus arteriosus. Nat Genet. 2006;38(3):343-349.

10. Wang L, et al. Mutations in Myosin light chain kinase cause familial aortic dissections. Am J Hum Genet. 2010;87(5):701-707.

11. Small EM, Olson EN. Pervasive roles of microRNAs in cardiovascular biology. Nature. 2011; 469(7330):336-342.

12. van Rooij E, et al. Dysregulation of microRNAs after myocardial infarction reveals a role of miR-29 in cardiac fibrosis. Proc Natl Acad Sci U S A. 2008; 105(35):13027-13032.

13. Ott CE, et al. MicroRNAs differentially expressed in postnatal aortic development downregulate elastin via $3^{\prime}$ UTR and coding-sequence binding sites. PLoS ONE. 2011;6(1):e16250.

14. Boon RA, et al. MicroRNA-29 in aortic dilation: implications for aneurysm formation. Circ Res. 2011;109(10):1115-1119.

15. Maegdefessel L, et al. Inhibition of microRNA-29b reduces murine abdominal aortic aneurysm development. J Clin Invest. 2012;122(2):497-506.

16. Kwiecinski $M$, et al. Hepatocyte growth factor (HGF) inhibits collagen I and IV synthesis in hepatic stellate cells by miRNA-29 induction. PLoS One. 2011;6(9):e24568.

17. Wang B, et al. Suppression of microRNA-29 expression by TGF-beta 1 promotes collagen expression and renal fibrosis [published online ahead of print November 17, 2011]. J Am Soc Nephrol. doi:10.1681/ ASN.2011010055.

18. Maurer B, et al. MicroRNA-29, a key regulator of collagen expression in systemic sclerosis. Arthritis Rheum. 2010;62(6):1733-1743.

19. Ogawa T, Iizuka M, Sekiya Y, Yoshizato K, Ikeda K, Kawada N. Suppression of type I collagen production by microRNA-29b in cultured human stellate cells. Biochem Biophys Res Commun. 2010;391(1):316-321.

20. Qin W, et al. TGF-beta/Smad3 signaling promotes renal fibrosis by inhibiting miR-29. J Am Soc Nephrol. 2011;22(8):1462-1474.

21. Daugherty A, Cassis LA. Mouse models of abdomi- nal aortic aneurysms. Arterioscler Thromb Vasc Biol. 2004;24(3):429-434.

22. Hanada K, et al. Perturbations of vascular homeostasis and aortic valve abnormalities in fibulin-4 deficient mice. Circ Res. 2007;100(5):738-746.

23. Wang Y, et al. TGF-beta activity protects against inflammatory aortic aneurysm progression and complications in angiotensin II-infused mice. J Clin Invest. 2010;120(2):422-432.

24. Gomez D, et al. Syndromic and non-syndromic aneurysms of the human ascending aorta share activation of the Smad2 pathway. J Pathol. 2009;218(1):131-142.

25. Inamoto $S$, et al. TGFBR2 Mutations alter smooth muscle cell phenotype and predispose to thoracic aortic aneurysms and dissections. Cardiovasc Res. 2010;88(3):520-529.

26. Tieu BC, et al. Aortic adventitial fibroblasts participate in angiotensin-induced vascular wall inflammation and remodeling. J Vasc Res. 2011; 48(3):261-272.

27. Wang H, et al. NF-kappaB-YY1-miR-29 regulatory circuitry in skeletal myogenesis and rhabdomyosarcoma. Cancer Cell. 2008;14(5):369-381.

28. Hu Y, et al. Abundant progenitor cells in the adventitia contribute to atherosclerosis of vein grafts in ApoE-deficient mice. J Clin Invest. 2004; 113(9):1258-1265.

\title{
Preserving postischemic reperfusion in the kidney: a role for extracellular adenosine
}

\author{
Joel M. Weinberg ${ }^{1}$ and Manjeri A. Venkatachalam² \\ 1Division of Nephrology, Department of Internal Medicine, Veterans Affairs Ann Arbor Healthcare System and University of Michigan, Ann Arbor, \\ Michigan, USA. ${ }^{2}$ Department of Pathology, University of Texas Health Science Center (UTHSC) at San Antonio, San Antonio, Texas, USA.
}

\begin{abstract}
Several adenosine receptor subtypes on endothelial, epithelial, mesangial, and inflammatory cells have been implicated in ischemic acute kidney injury, a life-threatening condition that frequently complicates the care of hospitalized patients. In this issue of the JCI, Grenz and coworkers provide novel insight into how preservation of postischemic renal perfusion by endothelial cell adenosine A2B receptors is antagonized by adenosine reuptake into proximal tubule cells by equilibrative nucleotide transporter 1 , which can be inhibited by dipyridamole. The work suggests that adenosine A2B receptor agonists and inhibition of equilibrative nucleoside transporters by dipyridamole may have therapeutic potential in ischemic acute kidney injury, a condition for which there are currently no specific therapeutic interventions.
\end{abstract}

Acute kidney injury (AKI) is a life-threatening condition that frequently complicates the care of hospitalized patients. It is clinically defined by an abrupt reduction in renal clearance function (as typically measured by an increase in serum creatinine) and, when associated with structural damage to the renal parenchyma, can be prolonged, require dialysis support prior to recovery,

Conflict of interest: The authors have declared that no conflict of interest exists.

Citation for this article: J Clin Invest. 2012; 122(2):493-496. doi:10.1172/JCI60957. and eventuate in chronic kidney disease even if recovery from the acute insult occurs $(1,2)$. While AKI can have multiple etiologies, ischemia is common to many of them (1) and predictably occurs in settings such as coronary bypass, where up to $7 \%-10 \%$ of patients develop AKI, which has been associated with $40 \%-70 \%$ mortality in the smaller cohort that requires dialysis and with increased subsequent long-term mortality even if dialysis is not needed (3).

Despite intensive research efforts and identification of multiple approaches effective in experimental models, there are no specific therapeutic interventions for treatment of AKI other than renal replacement therapy. Several targeted therapies have been tested in the clinic, but thus far all have failed (4). Both a better understanding of the underlying pathogenesis and new therapeutic targets continue to be needed. In this issue of the JCI, Grenz et al. elucidate a role for the purine nucleoside adenosine in protecting mouse kidneys from ischemic AKI by preserving postischemic blood flow (5). If the phenomena observed in mice hold true in humans, these data have important therapeutic implications, as they suggest that modulating adenosine levels via effects on adenosine $A 2 B$ receptor (Adora2b) on endothelial cells might be of benefit in individuals with AKI.

\section{Generation of adenosine during AKI} and its multiple potential effects Changes in purine nucleotide metabolism are a hallmark of tissue oxygen deprivation (6). Within cells, failure to maintain ATP production due to limitation of oxidative phosphorylation and compensatory 
anaerobic glycolysis leads to the degradation of ATP, eventually to its nucleoside components. Both purine nucleotides and nucleosides are released from tissue parenchymal cells and circulating cells to the extracellular space, where they activate cell surface receptors $(7,8)$ and can undergo further metabolism by cell surface enzymes that convert ATP and ADP to AMP (9) and then AMP to adenosine (8). Extracellular adenosine modulates multiple physiological processes. Its effects are mediated via four distinct $G$ protein-coupled receptors, the $\mathrm{A} 1$ adenosine receptor (Adora1), the $\mathrm{A} 2 \mathrm{~A}$ adenosine receptor (Adora2a), Adora2b, and the $\mathrm{A} 3$ adenosine receptor (Adora3). Activation of several of these receptors has been shown to play a major role in the response to injury in multiple tissues, including the response to oxygen deprivation (7).

In the kidney, adenosine produced by epithelial cells of the macula densa, a group of modified epithelial cells in the distal convoluted tubule, in response to solute delivery is centrally involved in the regulation of glomerular filtration rate (GFR) $(10,11)$. The net effect of adenosine in this process is to decrease GFR, primarily by stimulating vasoconstriction of afferent arterioles by activation of Adora1 on extraglomerular mesangial cells $(10,11)$, but recent work also suggests a contribution to the decreased GFR of vasodilation produced by efferent arteriolar Adora2b (12). Promotion and amelioration of AKI through the effects of adenosine on several of its receptors have been reported. Adora1-mediated afferent arteriolar vasoconstriction has been implicated in the suppression of GFR during AKI (10), but protective effects of activation of Adora1 on epithelial cells have also been documented (13). Adora2a on both neutrophils and $\mathrm{T}$ cells has potent, protective, anti-inflammatory effects (14). Adora2b has been shown to be necessary for ischemic preconditioning in the kidney (15).

\section{Modulation of endothelial Adora2b activation by proximal tubule adenosine transport}

In this issue of the JCI, Grenz et al. elegantly and comprehensively reveal a new dimension to the role of adenosine during AKI by showing that adenosine can protect from ischemic AKI in the mouse by preserving peritubular capillary blood flow during reperfusion (5). They find that adenosine mediates this effect by activating Adora2b on endothelial cells, which is opposed by adenosine uptake by equilibrative nucleoside transporter 1 (Ent1) at the basolateral surface of proximal tubules (Figure 1).

Grenz et al. used multiple pharmacological and genetic approaches to convincingly demonstrate an effect of this pathway in their model that exceeds the effects of other reported actions of adenosine during AKI. Treating mice with dipyridamole, a nonspecific Ent inhibitor, prior to initiation of ischemic AKI increased extracellular levels of adenosine in the kidney and protected against the functional and structural changes caused by ischemia. The benefit of dipyridamole was abolished by selective deletion of the gene encoding Adora $2 b$ in vascular endothelial cells, but not by deleting Adora1, Adora2a, or Adora3. Deletion of Adora2b in proximal tubule cells failed to abolish the protective effects of dipyridamole. Increasing the availability of extracellular adenosine by deleting the gene encoding Ent1 also was protective. Deleting Ent2 had no effect. The beneficial effect of Ent1 deletion could be reversed by kidney-specific lentiviral transduction with human ENT1. Consistent with involvement of tubule cell Ent1, studies with chimeric mice localized the effects of Ent 1 to radiation-resistant tissue cells rather than hematopoietic cells. Kidneys protected by adenosine activation of Adora $2 \mathrm{~b}$ displayed less tissue hypoxia and strikingly improved recovery of renal blood flow by ultrasound and intravital microscopy in the immediate postischemic period, which appears to largely account for the benefit observed.

\section{Sources of adenosine for Adora2b activation}

An interesting question raised by the work of Grenz et al. (5) is, what is the source of the adenosine? The endothelial cells themselves, tubule epithelial cells, and intravascular leukocytes and platelets are all possibilities (refs. 7, 8, 10, and Figure 1). The pool of nucleotides in tubule epithelial cells is particularly large because of the relative mass of those cells, and intact nucleotides can leak from these cells to the extracellular space (16), likely via connexin hemichannels that open during ischemia (17). Upon leakage from tubule epithelial cells, extracellular AMP can be converted to adenosine by the ecto-5'-nucleotidase CD73, which is highly expressed on interstitial pericytes, but not on the peritubular capillary endothelium (18). Metabolism of extracellular ATP and ADP requires the ecto-nucleoside $5^{\prime}$-triphosphate diphos- phohydrolase CD39, which is not found on pericytes but is present on the peritubular capillary endothelium (5). Alternatively, adenosine formed within tubule epithelial cells could exit via Ent 1 or other pathways during ischemia. Adenosine could also derive from endothelial cells themselves or from intravascular neutrophils or platelets via release of ATP followed by its sequential metabolism via CD39 and CD73, both of which are present on neutrophils (7). Irrespective of the source, the data provided by Grenz et al. (5) are clear in showing that Ent1 in the tubule basolateral membrane effectively competes for adenosine with Adora2b to almost completely prevent Adora2b activation. Polyethylene glycol-modified adenosine deaminase, which should initially be confined to the vascular space, also completely prevented the protective effect of increasing extracellular levels of adenosine with dipyridamole (5). Thus, adenosine availability to endothelial Adora2b is sensitively regulated by both intravascular and extravascular processes that decrease its concentration.

Tubule cells use both concentrative and equilibrative nucleoside transporters for uptake of nucleosides $(10,19)$. However, the concentrative transporters are in the apical brush border membrane, so they do not impact the adenosine movements relevant for the regulation of Adora2b activity elucidated by Grenz et al. (5). Adenosine reuptake from the extracellular space by Ent 1 is potentially desirable for epithelial metabolic recovery following reperfusion because restoration of intracellular adenine nucleotides from nucleosides is energetically more favorable than de novo synthesis and is more effective from adenosine than from inosine or subsequent metabolites (19). The data provided by Grenz et al. (5), however, show that this adenosine reuptake opposes Adora2b-mediated protection of the endothelium and promotion of reperfusion and the latter effects are most important for the overall tissue response. The downregulation of Ent1 by ischemia and hypoxia also described by Grenz et al. (5) would favor these vascular protective effects of adenosine.

\section{Role of Adora2b as opposed to other adenosine receptors}

Another notable aspect of the work of Grenz et al. (5) is the complete dependence of the benefit of increasing adenosine either using dipyridamole or through Ent1 deletion on the presence of Adora2b, which 


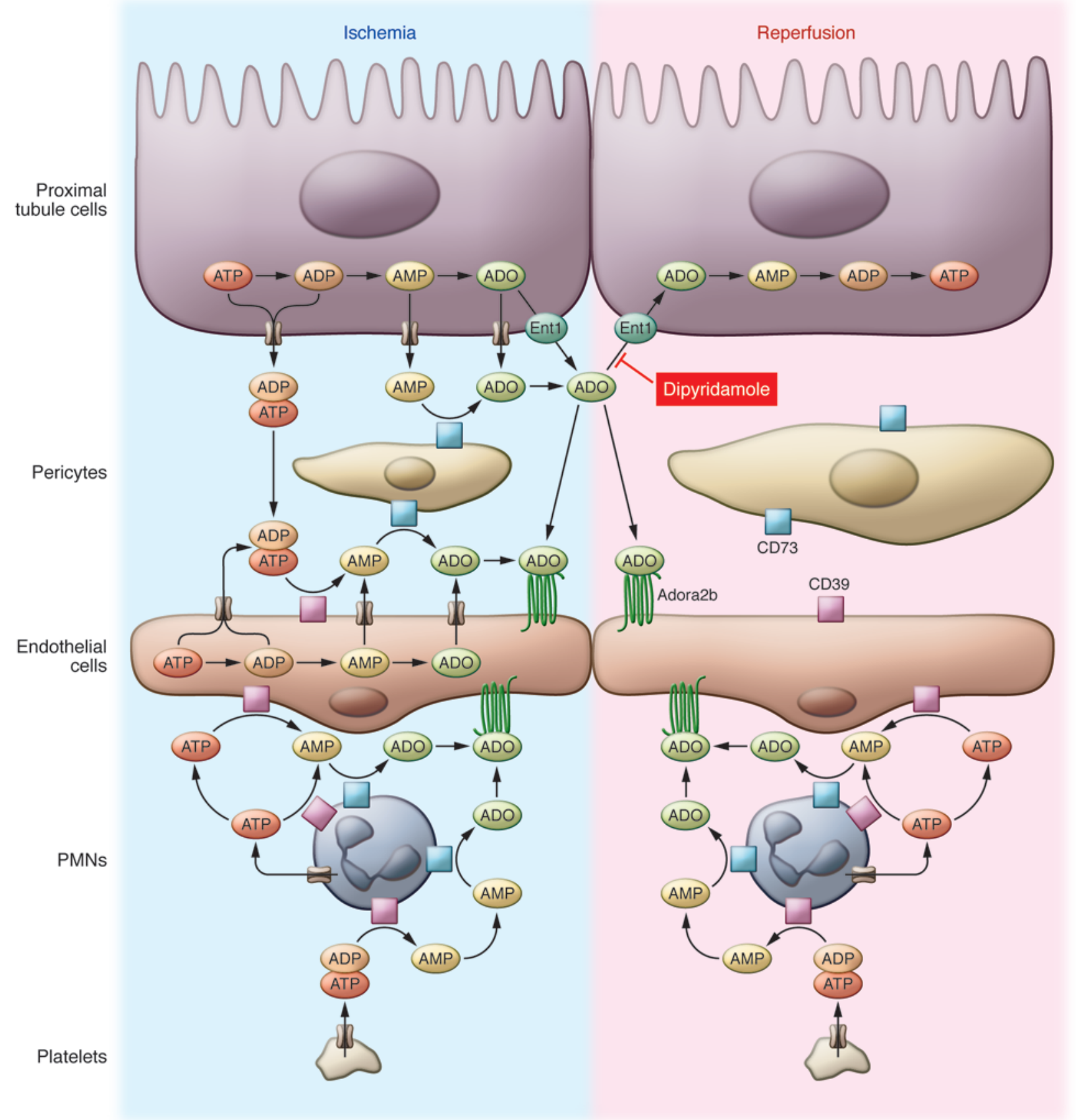

\section{Figure 1}

Sites of interacting processes in Adora2b-mediated protection against AKI. During ischemia, adenine nucleotides and adenosine can theoretically be released from tubule cells, endothelial cells, leukocytes, or platelets. Released ATP and ADP can be converted to AMP by CD39 on either endothelial cells or leukocytes (polymorphonuclear leukocytes [PMNs]). The released AMP can be further metabolized to adenosine by CD73, which in the kidney is most highly expressed on pericytes. CD73 is also expressed on leukocytes. In this issue of the JCl, Grenz et al. clearly show using mutant mice that activation by adenosine of Adora2b localized to the endothelium can preserve peritubular capillary blood flow that protects the tissue during reperfusion (5). Normally, despite the multiple potential extravascular and intravascular sites of adenosine generation, endothelial Adora2b activation is largely prevented by adenosine reuptake into proximal tubule cells by Ent1. Dipyridamole blocks Ent1-mediated adenosine reuptake and enables protective Adora2b activation. The large mass of proximal tubule cells as a source of released nucleotides and adenosine, the positioning of CD73 on pericytes to generate adenosine from nucleotides released by the tubules, and the sensitivity of adenosine availability to tubule cell Ent1 suggest that tubular cell adenosine generation is more important than intravascular for Adora2b activation. $\mathrm{ADO}$, adenosine. 
would seem inconsistent with the antiinflammatory effects of adenosine mediated by Adora2a (14) and the well-documented protective effects during AKI that have also been demonstrated for Adora1 in the mouse (13). Maintaining higher adenosine levels with dipyridamole or Ent1 deletion should also have promoted the latter two pathways. Grenz et al. attribute the apparent lack of effect on Adora1- and Adora2amediated pathways to the higher affinity of those receptors for adenosine (5), which allows their activation at lower adenosine levels than the levels reached with dipyridamole and Ent1 deletion and needed for activation of Adora2b. The Adora2b-mediated effects described by Grenz et al. clearly most strongly impact the immediate reflow period, and they tested dipyridamole and the Adora2b agonist PSB1115 only as a pretreatment or just before reflow. They did not test administration at later points. Promoting the anti-inflammatory effects of Adora2a on neutrophils and $T$ cells (14) or the protective effects of Adora1 on parenchymal cells (13) could provide more possibilities for delayed interventions in the many translational settings in which pretreatment is not feasible. It is also conceivable that delayed activation of Adora $2 b$ after early reflow could be deleterious, for example, through proinflammatory stimulation of dendritic cells (20).

The strong benefit of dipyridamole reported by Grenz et al. (5) differs from previous work in the rat, where dipyridamole was shown to aggravate clamp ischemia-induced AKI (21). These divergent observations may relate to species differences or could be secondary to the mode of induction of ischemia. For most of their studies, Grenz et al. used a sling to constrict the renal artery, which appears to give highly reproducible results, but in this approach, the artery may occlude less completely than with the clamps used in the rat study and most other mouse work, so the insult in these other models may be more severe. In supplemental data, Grenz et al. (5) report work in which they induced a 20-minute insult using a bilateral clamp model of ischemic AKI and showed beneficial effects of dipyridamole and Ent1 knockout in terms of early recovery of GFR and improved histology, which could mean that longer durations are problematic and the benefit only occurs in a limited window of time.

\section{Relevance to human AKI}

The impaired postischemic reflow shown by Grenz et al. (5) to be alleviated by Ent1sensitive Adora2b activation is well established to play a major role in the injury caused in small animal ischemia/reperfusion models (22-25). For technical reasons, Grenz et al. measured capillary perfusion in the superficial cortex, but it is more severely impaired in the deep cortex and outer medulla, where most structural damage develops (22-25). The authors have done service to the field by placing the complexities of adenosine transport and signaling in the context of reperfusion abnormalities. The translational relevance of their findings will depend on the role of reperfusion abnormalities during human AKI, which, as Grenz et al. point out, is largely unknown. Other maneuvers that have increased renal perfusion and alleviated AKI in small animal models, such as treatment with atrial natriuretic peptide, have failed in human trials (4). The relatively larger and more elongated medulla of rats and mice may predispose them to ischemic damage during reperfusion as compared with other species such as the dog and the human (22). Nonetheless, the striking nature of the findings in the work by Grenz et al., the clear delineation of the mechanism involved, and the availability of pharmacologic approaches that will be suitable for human application make translational testing of these findings appealing, particularly in settings where the insult can be anticipated and pretreatment is possible.

\section{Acknowledgments}

This work was supported by NIH grant DK-34275.

Address correspondence to: Joel M. Weinberg, Nephrology Division, Department of Internal Medicine, Room 1560, MSRB II, University of Michigan Medical Center, Ann Arbor, Michigan 48109-0676, USA. Phone: 734.764.3157; Fax: 734.763.0982; E-mail:wnberg@umich.edu.

1. Thadhani R, Pascual M, Bonventre JV. Medical progress - acute renal failure. $N$ Engl J Med. 1996;334(22):1448-1460.

2. Venkatachalam MA, Griffin KA, Lan R, Geng H, Saikumar P, Bidani AK. Acute kidney injury: a springboard for progression in chronic kidney disease. Am J Physiol Renal Physiol. 2010;298(5):F1078-F1094.

3. Rosner MH, Portilla D, Okusa MD. Cardiac surgery as a cause of acute kidney injury: pathogenesis and potential therapies. Intensive Care Med. 2008;23(1):3-18.
4. Jo SK, Rosner MH, Okusa MD. Pharmacologic treatment of acute kidney injury: why drugs haven't worked and what is on the horizon. Clin J Am Soc Nephrol. 2007;2(2):356-365.

5. Grenz A, et al. Equilibrative nucleoside transporter 1 (ENT1) regulates postischemic blood flow during acute kidney injury in mice. $J$ Clin Invest. 2012;122(2):693-710.

6. Weinberg JM. The cell biology of ischemic renal injury. Kidney Int. 1991;39(3):476-500.

7. Grenz A, Homann D, Eltzschig HK. Extracellular adenosine: a safety signal that dampens hypoxiainduced inflammation during ischemia. Antioxid Redox Signal. 2011;15(8):2221-2234.

8. Vallon V, Rieg T. Regulation of renal $\mathrm{NaCl}$ and water transport by the ATP/UTP/P2Y(2) receptor system. Am J Physiol Renal Physiol. 2011; 301(3):F463-F475.

9. Kishore BK, et al. Expression of NTPDase1 and NTPDase2 in murine kidney: relevance to regulation of $\mathrm{P} 2$ receptor signaling. Am J Physiol Renal Physiol. 2005;288(5):F1032-F1043.

10. Vallon V, Muhlbauer B, Osswald H. Adenosine and kidney function. Physiol Rev. 2006;86(3):901-940.

11. Castrop H, et al. Impairment of tubuloglomerular feedback regulation of GFR in ecto-5'nucleotidase/CD73-deficient mice. J Clin Invest. 2004;114(5):634-642.

12. Al-Mashhadi RH, Skott O, Vanhoutte PM, Hansen $\mathrm{PB}$. Activation of $\mathrm{A}(2)$ adenosine receptors dilates cortical efferent arterioles in mouse. Kidney Int. 2009;75(8):793-799.

13. Kim M, et al. Kidney-specific reconstitution of the A1 adenosine receptor in A1 adenosine receptor knockout mice reduces renal ischemia-reperfusion injury. Kidney Int. 2009;75(8):809-823.

14. Laubach VE, French BA, Okusa MD. Targeting of adenosine receptors in ischemia-reperfusion injury. Expert Opin Ther Targets. 2011;15(1):103-118.

15. Grenz A, et al. The reno-vascular A2B adenosine receptor protects the kidney from ischemia. PLoS Med. 2008;5(6):e137.

16. Weinberg JM. Adenine nucleotide metabolism by isolated kidney tubules during oxygen deprivation. Biochem Med Metab Biol. 1988;39(3):319-329.

17. Hanner F, Sorensen CM, Holstein-Rathlou NH, PetiPeterdi J. Connexins and the kidney. Am J Physiol Regul Integr Comp Physiol. 2010;298(5):R1143-R1155.

18. Lehir M, Kaissling B. Distribution and regulation of renal ecto-5'-nucleotidase: implications for physiological functions of adenosine. Am J Physiol. 1993;264(3 pt 2):F377-F387.

19. Weinberg JM, Davis JA, Lawton A, Abarzua M. Modulation of cell nucleotide levels of isolated kidney tubules. Am J Physiol. 1988;254(3 pt 2):F311-F322.

20. Wilson JM, et al. The A(2B) The A2B adenosine receptor promotes $\mathrm{Th} 17$ differentiation via stimulation of dendritic cell IL-6. J Immunol. 2011;186(12):6746-6752.

21. Lin JJ, Churchill PC, Bidani AK. The effect of dipyridamole on the initiation phase of postischemic acute renal failure in rats. Can J Physiol Pharmacol. 1987;65(7):1491-1495.

22. Lieberthal W, Nigam SK. Acute renal failure. I. Relative importance of proximal vs. distal tubular injury. Am J Physiol. 1998;275(5 pt 2):F623-F631.

23. Mason J, Torhorst J, Welsch J. Role of the medullary perfusion defect in the pathogenesis of ischemic renal failure. Kidney Int. 1984;26(3):283-293.

24. Brodsky SV, et al. Endothelial dysfunction in ischemic acute renal failure: rescue by transplanted endothelial cells. Am J Physiol Renal Physiol. 2002;282(6):F1140-F1149.

25. Sutton TA, Fisher CJ, Molitoris BA. Microvascular endothelial injury and dysfunction during ischemic acute renal failure. Kidney Int. 2002;62(5):1539-1549. 